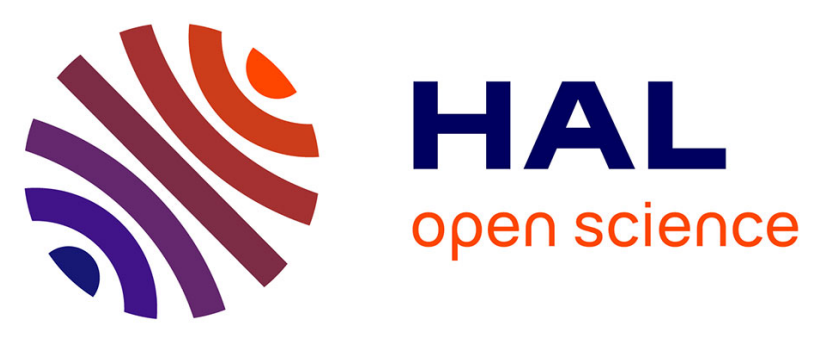

\title{
Measurement of mass exchange processes and coefficients in a simplified open-channel lateral cavity connected to a main stream
}

Emmanuel Mignot, Wei Cai, Juan Ignacio Polanco, Cristian Escauriaza, Nicolas Rivière

\section{To cite this version:}

Emmanuel Mignot, Wei Cai, Juan Ignacio Polanco, Cristian Escauriaza, Nicolas Rivière. Measurement of mass exchange processes and coefficients in a simplified open-channel lateral cavity connected to a main stream. Environmental Fluid Mechanics, 2017, 17, pp.429-448. 10.1007/s10652-016-9495-7 . hal-01724973

\section{HAL Id: hal-01724973 \\ https://hal.science/hal-01724973}

Submitted on 21 Nov 2018

HAL is a multi-disciplinary open access archive for the deposit and dissemination of scientific research documents, whether they are published or not. The documents may come from teaching and research institutions in France or abroad, or from public or private research centers.
L'archive ouverte pluridisciplinaire HAL, est destinée au dépôt et à la diffusion de documents scientifiques de niveau recherche, publiés ou non, émanant des établissements d'enseignement et de recherche français ou étrangers, des laboratoires publics ou privés. 


\title{
Measurement of mass exchange processes and coefficients in a simplified open-channel lateral cavity connected to a main stream
}

\author{
Emmanuel Mignot ${ }^{1}$, Wei Cai ${ }^{1}$, Juan Ignacio Polanco ${ }^{2}$, Cristian Escauriaza ${ }^{2}$ \\ and Nicolas Riviere ${ }^{1}$
}

Informative title: Mass exchange in a lateral cavity

${ }^{1}$ Univ Lyon, INSA Lyon, CNRS, LMFA UMR5509, F-69621 Villeurbanne, France.

${ }^{2}$ Departamento de Ingeniería Hidráulica y Ambiental. Pontificia Universidad Católica de

Chile, Av. Vicuña Mackenna 4860, 7820436 Santiago, Chile and Centro de Investigación

para la Gestion de Desastres Naturales (CIGIDEN)

* corresponding author: Emmanuel.mignot@insa-lyon.fr, 0033-472438070.

ABSTRACT: Lateral cavities are major storage zones in riverine environments for which the mass exchanges with the main stream strongly impact the characteristics of the habitat in these dead zones. An experimental work is presented here with a controlled main stream and a connected openchannel lateral cavity to assess the processes responsible for these exchanges and to quantify the exchange capacities. In a first step, the measurements of passive scalar transport allow us to identify the physical processes involved in the exchange of mass from the main stream and its spreading within the cavity. In a second step, the quantitative mass exchange coefficient, representative of the exchange capacity, is measured for 28 flow and cavity configurations. The sensibility analysis to the governing parameters proposed by the dimensional analysis then reveals that changing the geometric aspect ratio of the cavity does not affect the exchange coefficient while increasing the normalized water depth or decreasing the Reynolds number of the main stream tend to increase this coefficient. Indeed, these parameters modify both the growth rate of the mixing layer width at the interface and the amplitude of the alternating transverse velocity across the interface, thus affecting the exchange capacities from the main stream to the cavity.

KEYWORDS: Mass exchange, Turbulent diffusion, Cavity, Exchange coefficient, Dye release method

\section{INTRODUCTION}

Controlling the exchange of mass between a river and its connected storage zones is of primary importance for river management, given that in the storage zones the lower water velocities and 
higher local residence times enhance fish and vegetation biodiversity (Jackson et al., 2013). Moreover, sediment and dissolved contaminant transferred from the main stream to the transient storage zones can be accumulated and modified due to biogeochemical reactions (Ensign and Doyle, 2005; Argerich et al., 2011). Using former data from the literature, Jackson et al. (2013) proposed empirical relationships relating the mean residence time of dissolved material in a cavity on the field as a function of several non-dimensional parameters of the flow and storage zone characteristics. These measurements of exchange capacities or residence times can represent mass exchange processes in conditions with complex shapes and bathymetry of the cavity. Nevertheless, due to obvious experimental restrictions, these field experiments do not allow to i) Investigate in detail the physical processes involved in the mass introduction within the storage zone; and ii) Vary the flow and cavity parameters in a controlled way in order to identify the impact of all governing parameters. To overcome these limitations, the present paper is based on an experimental approach in controlled and simplified conditions in order to gain information on the physics of the mass exchange processes and the impact of the non-dimensional parameters on the exchange capacity. The selected storage zone geometry is an open-channel lateral cavity, which represents a major transient storage zone in riverine environments (Jackson et al., 2013).

Open-channel lateral cavities connected to rivers can be divided into (i) isolated cavities (as in the present case), (ii) cavities located between two consecutive groynes (as investigated by Uijttewaal et al., 2001 or Weitbrecht et al., 2008) and (iii) a series of lateral cavities (see Erpicum et al., 2009 or Meile et al., 2011). Differences between these configurations mainly lie on the impact of the cavities located upstream and downstream from the studied one, and particularly in the direction of the main stream when approaching the cavity: for case (i), the upstream flow is parallel to the sidewalls and is not affected by any upstream mixing layer; for case (iii), the mean flow is parallel to the side walls but the flow is affected by intense turbulent structures generated in the mixing layer of the upstream dead zone (see Tuna and Rockwell, 2014); finally for case (ii), the transverse component of the main stream is directed towards the center of the main stream. The present paper is dedicated to lateral cavities of type (i), such as oxbows, which are examples of natural isolated lateral cavities, or fluvial or sea harbors (respectively connected to rivers or sea streams), which are examples of artificial isolated lateral cavities.

It appears from the literature that the main parameter governing the flow pattern in an isolated lateral cavity is the aspect ratio $W / L$ of the cavity (see Mignot et al., 2017) where $L$ is the length of the cavity in the direction parallel to the main stream and $W$ is the cavity width along the crosswise direction, as sketched in Fig.1. Most studies in the literature consider a low aspect ratio $(W / L \leq 1)$ for which two main horizontal cells are aligned along the main stream axis for the lowest aspect ratios (Sanjou and Nezu, 2013) or a single cell occupies almost the whole available space in the cavity for 
aspect ratios approaching 1 (Mizumura and Yamasaka, 2002). For higher aspect ratios, Booij (1989) and Mignot et al. (2017) showed that for an aspect ratio $W / L$ equal to 3, a second stable cell takes place on the side of the first one, aligned along the width of the cavity $W$, in a similar way as for Lecoz et al. (2010) on a physical model with a very long curved cavity.

For all cases, at the interface between the main stream and the cavity, a large velocity gradient forms (Tuna et al., 2013) which leads to a horizontal mixing layer that extends from the upstream to the downstream corner of the cavity. Mignot et al. (2016) showed that the alternation of clockwise and counter-clockwise coherent turbulent structures, shed in the vicinity of the upstream corner and advected along the mixing layer towards the downstream corner, generates a periodic alternating transverse velocity across the interface. The mixing layer then transfers from the main stream towards the cavity: i) momentum, which maintains the recirculating motion of the flow in the cavity and ii) mass such as pollutants, nutrients and gases influencing the ecological equilibrium of the cavity, or fine sediments influencing the geomorphological budget.

The objectives of the present paper are, first, to gain information on the exchange processes of passive scalar such as pollutants between the main stream and the isolated open-channel lateral cavity and, second, to identify the main parameters influencing the exchange capacity. The first section describes the experimental set-up used in the present work and introduces the two methodologies available in the literature to quantify the exchange coefficient. This section then proposes a dimensional analysis applied to the mass exchange coefficient and summarizes the available data in the literature and the present experimental data set. The second and third sections are then devoted to the physical description of the exchange processes and the sensitivity analysis permitting to conclude on the parameters that influence the mass exchange capacity in the lateral cavity flow.

\section{Methodology and literature data review}

\subsection{Experimental set-up}

The experiments are performed in the channel intersection facility at the Laboratoire de Mécanique des Fluides et d'Acoustique at the Université de Lyon, France. The facility consists of a 4.9m long horizontal glass channel of $b=0.3 \mathrm{~m}$ wide rectangular shape section, which intersects a $2 \mathrm{~m}$ wide (along $W$ ) similar glass side channel of length $L=0.3 \mathrm{~m}$ (see Fig.1) forming a $90^{\circ}$ angle (see Mignot et al., 2013 for more details on the facility). The width of the cavity $W$ can be continuously modified by displacing the movable wall that closes the side channel as sketched in Fig.1. A honeycomb at the inlet of the upstream branch serves to stabilize and straighten the inlet flow and a downstream sharp crested weir is used to adjust the water depth $h$ in the cavity region. The main 
stream discharge $Q$ is measured within the pumping loop using a Promag 50 flowmeter (from Endress Hauser; accuracy $\pm 0.05 \mathrm{~L} / \mathrm{s})$. The axis system $(x, y)$ is centered on the upstream corner of the cavity (see Fig.1) with the $x$ axis parallel to the main stream axis and the $y$ axis oriented from the main stream to the cavity and the instantaneous velocity components $u$ and $v$ are defined along the $x$ and $y$ axes respectively. For all flow configurations listed in Table 1, a maximum oscillation amplitude of the water depth equal to $3.3 \%$ is recorded using five wave probes distributed within the cavity area; the present configurations is then considered as "without seiching" (see Tuna et al., 2013).

Two main methodologies are reported in the literature to estimate the mass exchange capacity between a main stream and a dead zone, which are replicated herein for the open-channel lateral cavity. The first methodology, named "dye release method", is based upon continuously releasing dye in the main stream and measuring its accumulation in the cavity. The second methodology, named "transverse velocity method", is based upon velocity measurements at the interface between the main stream and the cavity. Both methodologies derive from the 1D mass exchange model presented in the next section.

\section{$1.21 \mathrm{D}$ mass exchange model}

The theoretical background for the dye release method derives from the 1D mass exchange model proposed by Valentine \& Wood (1977) and Uijttewaal et al. (2001), which states that the mass of a passive scalar passing from the main stream towards the cavity evolves with time as a function of the exchanges across the interface between these two regions. This relation reads:

$$
\frac{d M}{d t}=S_{e x} V_{e x}\left(C_{M S}-C_{C}(t)\right)
$$

where $M$ is the mass of scalar located within the cavity at time $t, S_{\mathrm{ex}}=L h$ is the interface section between the main stream and the cavity (see Fig.1), $V_{e x}$ is the characteristic, time and space averaged, exchange velocity across the interface, $C_{M S}$ is the approaching main stream concentration (here it is uniform and steady) and $C_{C}\left[\mathrm{~kg} \cdot \mathrm{m}^{-3}\right]$ is the spatially averaged scalar concentration in the cavity at time $t$. Moreover, Valentine \& Wood (1977) propose to relate $V_{e x}$ to the bulk velocity of the main stream $U_{b}=Q /(b h)$ as

$V_{e x}=k U_{b}$

where $k$ is the so-called non-dimensional exchange coefficient. The mass of scalar in the cavity at a given time is given by 
where $W L h$ is the volume of water in the cavity (constant with time). Thus Eq. 1 becomes:

$$
\frac{d C_{c}(t)}{d t}=\frac{k U_{b}}{W}\left(C_{M S}-C_{C}(t)\right)
$$

Integrating Eq. 4 leads to an expression for the evolution of the concentration in time,

$$
C_{C}(t)=A e^{-\frac{k U_{b}}{W} t}+C_{M S}
$$

with $A$ being a constant. The initial concentration in the cavity is zero $C_{C}(t=0)=0$, and the concentration in the main stream $C_{M S}$ remains constant with time. Then $A=-C_{M S}$, and Eq. 5 can be written as follows:

$$
\frac{C_{M S}-C_{C}(t)}{C_{M S}}=e^{-\frac{k U_{b}}{W} t}
$$

The efficiency of the mass exchange is accounted for by the exchange coefficient $k$. Its value can be estimated from the time evolution of the concentration (Eq. 6) through the dye release method, as described in section 1.3. The coefficient $k$ can also be directly estimated from the transverse velocity measurement (Eq. 2), as described in section 1.4 below.

\subsection{Dye release method}

The dye release method consists on the following steps:

- measuring the time evolution of averaged dye concentration in the cavity $C_{C}(\mathrm{t})$

- plotting the evolution of the left-hand-side term of Eq. 6 as a function of time

- identifying the slope of the curve in order to estimate $k$ using Eq.6. This $k$ is noted $k_{d y e}$ for "dye release method". 

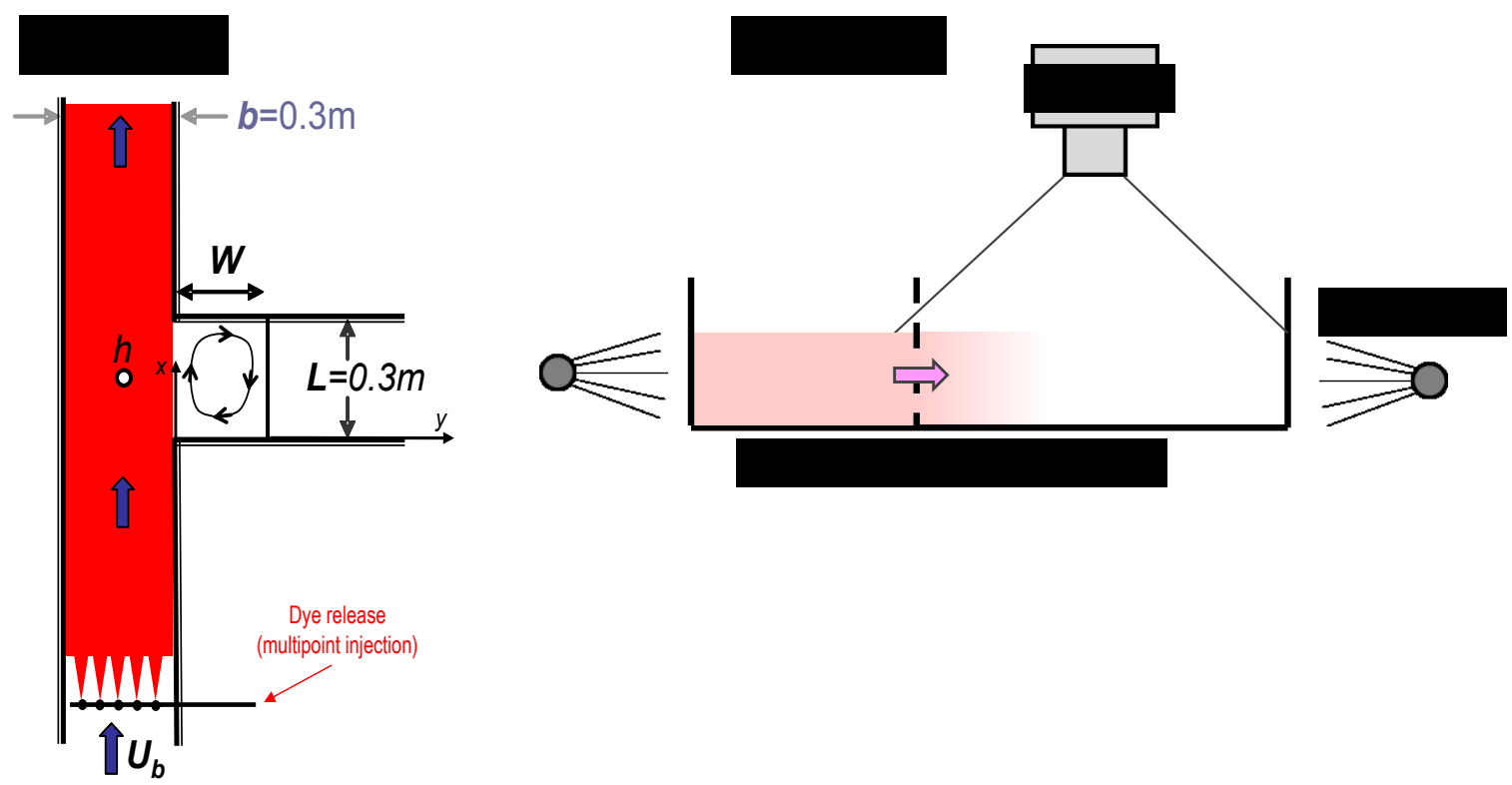

Figure 1. Scheme of the dye release method at initial time with a uniform concentration in the main stream and a null concentration in the cavity.

The whole set-up is installed inside of a dark tent where a controlled and repeatable ambient light is set using two broadband light sources as sketched on Fig. 1. Previous to performing the experiments, the cavity is closed and consecutively filled with volumes of water with various controlled dye concentrations so that the water depth remains exactly equal to the water depth of the studied flow configuration, as indicated in Table 1, and the lightning (light intensity and spatial orientation) is kept similar during the whole calibration process (see Weitbrecht et al., 2008). The dye used herein is red food color. A high-resolution photograph (4000-4000 pixels) of the cavity and intersection is taken from above the free surface for each concentration (see Fig. 1). A calibration curve of light intensity vs. dye concentration is then established for each pixel. Before starting the measurements, a steady-state clear-water flow is established in the main stream and the cavity. Then, a constant discharge of dye (two orders of magnitude lower than the water discharge) is injected at the upstream end of the inlet channel using a multipoint injection (see Fig.1) so that complete mixing is achieved in the upstream channel before reaching the cavity. The initial time $t=0$, is set when the dye reaches the intersection. Similar photos under similar lightning as in the calibration process are taken along time. The light intensity at each pixel of each photo, along with all the calibration curves finally allow to estimate the concentration map of $C_{\mathrm{C}}(x, y, t) / C_{\mathrm{MS}}$ at every time step (see Fig.2).

The major limitations of such approach for estimating $k$ (using Eq.6) are detailed hereafter. First the concentration field is assumed to be $2 \mathrm{D}$, i.e. with no evolution along the vertical axis so that the estimated concentration at one pixel on the CCD sensor of the camera, which corresponds to the concentration of the top layer of the corresponding water column, is representative of the whole 
water column. Actually, the higher the concentration, the more opaque the water volume and thus the thinner this top-layer "seen" by the camera. Nevertheless, the uncertainty of the method (at least as it is applied herein) is not limited to the vertical non-uniformity of concentration, but rather to the non-uniformity of the whole water volume. Indeed, the light intensity recorded by the CCD sensor relates the light attenuation along its whole path, from the light sources, across the water volume and the free surface and finally to the camera (see Fig. 1). This light intensity is then compared with the light intensity in the calibration images, which are taken in water volumes with uniform concentrations. The second limitation of the method is then that we compare light attenuation in non-uniform concentration water during the mass-exchange process with uniform concentration water during the calibration process.

\subsection{Transverse velocity method}

The transverse velocity method consists in estimating the time and space averaged transverse exchange velocity $V_{\text {ex }}$ from a velocity measurement and then computing the exchange coefficient using Eq.2. Weitbrecht et al. (2008) proposed to evaluate $V_{\text {ex }}$ based on the measurement of transverse velocity along the main stream-cavity interface as:

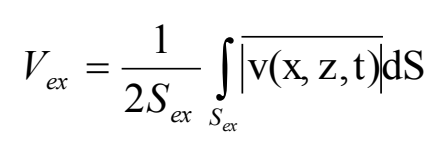

where $|v(x, z, t)|$ is the absolute value of the instantaneous transverse velocity component (along the $y$ axis, perpendicular to the main stream) and the overbar denotes time-averaging. The $k$ coefficient obtained through this method is noted $k_{\text {veloc }}$. Note that the value "2" in Eq. 7 derives from the fact that the absolute value of the transverse velocity component stands for the exchanges from the main stream to the cavity plus the exchanges from the cavity to the main stream, that is twice the exchanges represented by Eq.1.

The transverse velocity approach is performed here using 2D horizontal PIV data (as in Weitbrecht et al., 2008 or Tuna et al., 2013). A 40mW continuous laser coupled with a cylindrical lens generates a $1 \mathrm{~mm}$ thick horizontal light layer at the desired laser elevation $z_{L}$. Polyamid particles (diameter $50 \mu \mathrm{m}$ ) are added to the water. Finally, a video camera is located above the cavity interface in order to record the particle motion over 3000 consecutive images of 1280x960 pixels each with a frequency of $30 \mathrm{~Hz}$ and a spatial resolution of $0.3 \mathrm{~mm}$ per pixel (see Mignot et al., 2016 for more details). Then, the commercial software DaVis (from Lavision) allows to correct the image distortions, subtract the image background and finally compute the velocity field. It was verified that the maximum free-surface oscillations in the cavity remained negligibly small, with amplitudes smaller than $2 \%$ for all flows (lower than $0.5 \%$ for most flows). To estimate the uncertainty associated to the PIV measurements, we first verify that the Stokes number associated to the 
seeding particles remains low enough (St $\approx 0.05)$. Then, as in Mignot et al. (2014), the uncertainty associated to the measured velocity $\sigma_{\mathrm{v}}$ is estimated as that of the Gaussian peak fitting process, assumed to be equal to the velocity associated to a displacement of 0.1 pixel, plus that the seeding concentration gradient which is assumed to be equal to that of the Gaussian peak fitting (see Jahanmiri, 2011). This gives $\sigma_{v} \approx 2 \times 0.1$ pixel $\times 0.3 \mathrm{~mm} /$ pixel $\times 30 \mathrm{~Hz}=1.8 \mathrm{~mm} / \mathrm{s}$. Then the uncertainty associated to the exchange velocity $V_{e x}$ corresponds to $\sigma_{V e x}=\sigma_{\overline{|v|}} / 2=0.016 \mathrm{~mm} / \mathrm{s}$ where $\sigma_{\overline{|v|}}=\sigma_{|v|} / \sqrt{N}=\sigma_{v} / \sqrt{N}$ with $\mathrm{N}=3000$ velocity measurement samples in the present experiments. Assuming a 2D-flow (with no variation along $z$ ), Eq.7 can be simplified as:

$$
V_{e x 2}=\frac{1}{2 L} \int_{x=0}^{x=L} \overline{\left|\mathrm{v}\left(\mathrm{x}, \mathrm{z}=\mathrm{Z}_{\mathrm{L}}, \mathrm{t}\right)\right|} \mathrm{dx}
$$

and the exchange coefficient $k_{\text {veloc }}$ is finally obtained using Eq.2, using $V_{e x 2}$ instead of $V_{e x}$. Note that the 2D flow assumption is supported by the measurements performed by Tuna et al. (2013) and especially their figures 14 and 16 (left) without gravity standing waves, where the variation along $z$ of the time-averaged absolute value of transverse velocity remains limited.

\subsection{Discussion regarding the methods}

The dye release method is a physically-based method in the sense that it is effectively based on exchange measurements of a given passive scalar. However, the scalar concentration is estimated here from a camera located above the free surface, recording for each pixel the light intensity transmitted from the whole water column. Note that Sanjou and Nezu (2013) rather used LIF to measure the concentration field, with the limitation that their concentration is then estimated over a single horizontal plane. In both cases, $C_{C}$ (in Eq.6) is not a real volumetric averaged concentration but reduces to a concentration averaged over a portion of the cavity volume.

On the other hand, the transverse velocity method is simpler as it is based on velocity measurements; nevertheless, it is representative of the exchange processes only at early stages of mixing process, i.e. when the transfer is regulated by the dynamics of the shear layer. Tuna et al. (2013) revealed that an estimation of $V_{\text {ex }}$ through measurements at a single elevation in the upper part of the water column (assuming $V_{\mathrm{ex} 2} \sim V_{\mathrm{ex}}$ ) leads to a fair estimate of this coefficient. Moreover, Weitbrecht et al. (2008) obtained an average error of $17 \%$ between the two techniques in a groyne field cavity (see their table 2).

\subsection{Dimensional analysis and data set}

The dimensional analysis applied to the mass exchange coefficient $k$ in the present configuration is a simplification of the one proposed by Jackson et al. (2013) as it is here applied to a horizontal, rectangular, smooth cavity connected to a subcritical, straight, steady, smooth main stream of 
rectangular cross-section, with no step at the interface, and all walls in the cavity and the main stream being vertical. Moreover, assuming that the boundary layers are fully developed, this dimensional analysis finally involves 9 variables that are the cavity width $W$ and length $L$, the main stream width $b$, the roughness of the mainstream and cavity walls $k_{s}$, the bulk velocity of the main stream $U_{b}$, the water depth in the cavity and the mainstream $h$, the acceleration due to gravity $g$, and finally the fluid density $\rho$ and dynamic viscosity $\mu$. In the present case $b=L$, so that the number of variables reduces to 8 . Choosing as length scale $L$, as time scale $L / U_{\mathrm{b}}$ and as mass scale $\rho L^{3}$, the dimensional analysis reveals that 5 dimensionless parameters characterize the exchange coefficient, i.e:

$$
k=\mathrm{f}\left(W / L, \operatorname{Fr}, \operatorname{Re}, h / L, k_{\mathrm{s}} / L\right)
$$

where Re and Fr are the upstream Reynolds and Froude numbers of the main stream, respectively. The dimensionless roughness height $k_{S} / L$ can be discarded as, with glass walls, the flow regime in the main stream and the cavity is hydraulically smooth. Similarly, the effect of the Froude number $F r=U_{b} / \sqrt{g h}$ is discarded as only subcritical main streams are considered herein, with a Froude number that does not exceed 0.25 (see Table 1). This assumption that the Froude number has a negligible influence is supported by the recent work from Karimpour and Chu (2015). Through Direct Numerical Simulations, the authors showed (in their figure 5a) that the growth rate of the mixing layer width (see Figure 10) remains about constant for convective Froude numbers $\left(\operatorname{Fr}_{\mathrm{c}}\right)$ lower than 0.25 (and decreases for larger $\operatorname{Fr}_{\mathrm{c}}$ values), with $\mathrm{Fr}_{\mathrm{c}} \sim \mathrm{Fr} / 2$. As $\mathrm{Fr}<0.23$ in Table 1 (i.e. $\mathrm{Fr}_{\mathrm{c}} \leq 0.12$ ), the effect of variations of $\mathrm{Fr}$ on the characteristics of the mixing layer is assumed to be negligible. Note however that in realistic rivers, this Froude number can reach much larger values and thus should no longer be discarded from the dimensional analysis. The 3 remaining dimensionless parameters considered herein are then:

- $\quad W / L$ : the geometrical aspect ratio of the cavity

- $\quad h / L:$ the shallowness parameter

- $\quad$ Re: the Reynolds number of the main stream, defined as $\operatorname{Re}=4 \rho U_{b} L h /[\mu(L+2 h)]$.

Table 1 then comprises 3 series of experiments:

- in Series 1, the main stream flow conditions remain constant and only the aspect ratio of the cavity $W / L$ is varied, by regularly increasing its width $W$.

- in Series 2, the dimensions of the cavity remain unchanged, so does the water depth in the whole domain; the bulk velocity in the main stream is gradually increased, leading to a corresponding increase of the Reynolds number Re. 
- in Series 3, the dimensions of the cavity remain unchanged, so does the bulk velocity of the main stream; the water depth is gradually increased, leading to a corresponding increase of the normalized water depth $h / L$ and of the Reynolds number Re.

\begin{tabular}{|c|c|c|c|c|c|c|c|c|}
\hline & & & le 1. Al & $m e$ & $a$ & rat & & \\
\hline & $W(\mathbf{m})$ & $h(\mathbf{m})$ & $U_{b}(\mathrm{~m} / \mathrm{s})$ & $\boldsymbol{W} / \boldsymbol{L}$ & $h / L$ & Fr & $\operatorname{Re}\left(\mathrm{x}^{10^{4}}\right)$ & $\boldsymbol{k}_{\text {veloc }}$ \\
\hline \multirow{5}{*}{$\overrightarrow{\tilde{\sigma}}$} & 0.3 & 0.07 & 0.167 & 1 & 0.23 & 0.202 & 3.19 & 0.0252 \\
\hline & 0.6 & 0.07 & 0.167 & 2 & 0.23 & 0.202 & 3.19 & 0.0272 \\
\hline & 0.9 & 0.07 & 0.167 & 3 & 0.23 & 0.202 & 3.19 & 0.0275 \\
\hline & 1.2 & 0.07 & 0.167 & 4 & 0.23 & 0.202 & 3.19 & 0.0256 \\
\hline & 1.5 & 0.07 & 0.167 & 5 & 0.23 & 0.202 & 3.19 & 0.0255 \\
\hline \multirow{11}{*}{ 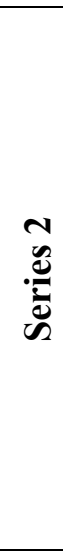 } & 0.3 & 0.07 & 0.071 & 1 & 0.23 & 0.086 & 1.36 & 0.0328 \\
\hline & 0.3 & 0.07 & 0.083 & 1 & 0.23 & 0.101 & 1.59 & 0.0309 \\
\hline & 0.3 & 0.07 & 0.095 & 1 & 0.23 & 0.115 & 1.82 & 0.0286 \\
\hline & 0.3 & 0.07 & 0.107 & 1 & 0.23 & 0.129 & 2.05 & 0.029 \\
\hline & 0.3 & 0.07 & 0.119 & 1 & 0.23 & 0.144 & 2.27 & 0.0295 \\
\hline & 0.3 & 0.07 & 0.131 & 1 & 0.23 & 0.158 & 2.50 & 0.0278 \\
\hline & 0.3 & 0.07 & 0.143 & 1 & 0.23 & 0.172 & 2.73 & 0.0267 \\
\hline & 0.3 & 0.07 & 0.155 & 1 & 0.23 & 0.187 & 2.95 & 0.0257 \\
\hline & 0.3 & 0.07 & 0.167 & 1 & 0.23 & 0.201 & 3.18 & 0.0258 \\
\hline & 0.3 & 0.07 & 0.179 & 1 & 0.23 & 0.215 & 3.41 & 0.026 \\
\hline & 0.3 & 0.07 & 0.190 & 1 & 0.23 & 0.230 & 3.64 & 0.0264 \\
\hline \multirow{12}{*}{ 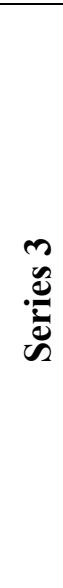 } & 0.3 & 0.03 & 0.119 & 1 & 0.10 & 0.219 & 1.19 & 0.0279 \\
\hline & 0.3 & 0.04 & 0.119 & 1 & 0.13 & 0.189 & 1.50 & 0.0258 \\
\hline & 0.3 & 0.05 & 0.119 & 1 & 0.17 & 0.169 & 1.79 & 0.0281 \\
\hline & 0.3 & 0.06 & 0.119 & 1 & 0.20 & 0.155 & 2.04 & 0.0263 \\
\hline & 0.3 & 0.07 & 0.119 & 1 & 0.23 & 0.143 & 2.27 & 0.0283 \\
\hline & 0.3 & 0.08 & 0.119 & 1 & 0.27 & 0.134 & 2.48 & 0.0276 \\
\hline & 0.3 & 0.09 & 0.119 & 1 & 0.30 & 0.126 & 2.68 & 0.029 \\
\hline & 0.3 & 0.10 & 0.119 & 1 & 0.33 & 0.120 & 2.86 & 0.0276 \\
\hline & 0.3 & 0.11 & 0.119 & 1 & 0.37 & 0.114 & 3.02 & 0.0293 \\
\hline & 0.3 & 0.12 & 0.119 & 1 & 0.40 & 0.109 & 3.17 & 0.0304 \\
\hline & 0.3 & 0.13 & 0.119 & 1 & 0.43 & 0.105 & 3.32 & 0.0305 \\
\hline & 0.3 & 0.14 & 0.119 & 1 & 0.47 & 0.101 & 3.45 & 0.0304 \\
\hline
\end{tabular}

\subsection{Data from the literature}

In the literature, the exchange coefficient $k$ between a main stream and a dead zone in laboratory conditions was measured for various configurations such as a groyne field (see for instance Uijttewaal et al., 2001 and Weitbrecht et al., 2008), a closed cavity located below the main flow (as for Caton et al., 2003), a series of closed cavities below the main flow, or "street canyons" (as Salizzoni et al., 2009 or Markides et al., 2010) and finally a single lateral cavity as in the present configuration (corresponding references are detailed below).

Regarding studies of mass exchange in open-channel, single lateral cavities, about half of them exhibit specific geometrical or flow details that prohibit a direct comparison: Li \& Ip (1999) and Li 
\& Gu (2001) respectively measured and computed the mass exchange coefficient $k$ in a lateral cavity with an interface section $S_{\mathrm{ex}}$ (see Eq.1) limited by a partial wall; Langendoen et al. (1994) considered an oscillating inflow; Muto et al. (2000) included a step at the entrance of the lateral cavity; Jackson et al. (2013) considered cavities with complex rough bathymetries and Jackson et al. (2015) considered a semi-circular and two conic-shaped cavities. These studies are then excluded from the data used for comparison in Table 2.

The remaining simple lateral cavity configurations (horizontal and rectangular isolated openchannel lateral cavity, with a steady main stream and no wall or step at the cavity entrance) available in the literature correspond to four studies, included in Table 2, and detailed below.

First, Booij (1989) used the temperature as an indicator to estimate $k$ applying the "dye release method" for two flow configurations. However the temperature measurements were limited to a single location in the cavity so that $C_{C}$ (in Eq.6) was not spatially averaged.

Second, Altai \& Chu (1997) applied the dye release method by initially inserting dye in the cavity zone and measuring its concentration decay. No information is however given regarding the method used to estimate the dye concentration from the recorded images. These authors considered two square cavity dimensions with $W / L=1$ in both cases. For one cavity, they increased the main stream velocity, while for the other cavity they modified both the main stream velocity and water depth (see Table 2). However, the authors encountered no clear tendency of the $k$ coefficient with these parameters.

Third, Sanjou \& Nezu (2013) considered a narrow cavity with $W / L=1 / 3$ and applied the LIF method to measure the concentration field at one elevation near the surface region $(z / h=0.94)$ for a single flow configuration. In this case, $C c$ is then averaged only over a single measured horizontal plane. Fourth, Tuna et al. (2013) measured $k_{\text {veloc }}$ using PIV at 6 elevations for two flows: one without and one with seiching. They obtained two different $k_{\text {veloc }}$ profiles: from 0.026 (near the surface) to 0.03 (near the bed) without seiching and from 0.034 (near the surface) to 0.042 (at mid-elevation) with seiching (see Table 2).

The collected data from the literature does not permit to conclude regarding the impact of the dimensionless parameters sorted from the dimensional analysis (Eq. 9) on the mass exchange coefficient. The absence of a tendency is the starting motivation of the present work detailed in the next sections.

Table 2. Flow and geometrical configurations along with $k$ estimations obtained from the literature data review. 


\begin{tabular}{|c|c|c|c|c|c|c|c|c|c|c|}
\hline & \\
\hline & & $W(\mathrm{~m})$ & $L(\mathrm{~m})$ & $U_{b}(\mathrm{~m} / \mathrm{s})$ & $h(\mathrm{~m})$ & $W / L$ & $h / L$ & $\mathrm{Fr}$ & $\boldsymbol{k}_{\text {veloc }}$ & $\boldsymbol{k}_{\text {dye }}$ \\
\hline \multirow{3}{*}{$\begin{array}{l}\text { Tuna } \\
\text { et al. } \\
(2013)\end{array}$} & \multirow{3}{*}{$\begin{array}{c}\text { near-bed region: } \\
\text { surface region: } \\
\text { surface region - seiching: }\end{array}$} & 0.457 & 0.305 & 0.24 & 0.0381 & 1.5 & 0.125 & 0.63 & \multirow{3}{*}{$\begin{array}{l}0.030 \\
0.026 \\
0.035\end{array}$} & \\
\hline & & 0.457 & 0.305 & 0.24 & 0.0381 & 1.5 & 0.125 & 0.63 & & \\
\hline & & 0.457 & 0.305 & 0.43 & 0.0381 & 1.5 & 0.125 & 1.16 & & \\
\hline \multirow{9}{*}{\multicolumn{2}{|c|}{ Altai et Chu (1997) }} & 0.89 & 0.89 & 0.29 & 0.029 & 1 & 0.033 & 1.04 & & 0.028 \\
\hline & & 0.89 & 0.89 & 0.16 & 0.05 & 1 & 0.056 & 0.32 & & 0.014 \\
\hline & & 0.89 & 0.89 & 0.30 & 0.027 & 1 & 0.030 & 1.13 & & 0.025 \\
\hline & & 0.89 & 0.89 & 0.13 & 0.06 & 1 & 0.067 & 0.21 & & 0.019 \\
\hline & & 0.32 & 0.32 & 0.34 & 0.025 & 1 & 0.078 & 1.37 & & 0.028 \\
\hline & & 0.32 & 0.32 & 0.30 & 0.025 & 1 & 0.078 & 1.21 & & 0.014 \\
\hline & & 0.32 & 0.32 & 0.21 & 0.025 & 1 & 0.078 & 0.86 & & 0.019 \\
\hline & & 0.32 & 0.32 & 0.14 & 0.025 & 1 & 0.078 & 0.58 & & 0.013 \\
\hline & & 0.32 & 0.32 & 0.07 & 0.025 & 1 & 0.078 & 0.28 & & 0.020 \\
\hline \multirow{3}{*}{\multicolumn{2}{|c|}{ Booij (1989) }} & 1 & 1 & 0.50 & 0.11 & 1 & 0.110 & 0.46 & & 0.020 \\
\hline & & 3 & 1 & 0.50 & 0.11 & 3 & 0.110 & 0.46 & & 0.020 \\
\hline & & 3 & 1 & 0.50 & 0.11 & 3 & 0.110 & 0.46 & & 0.014 \\
\hline \multicolumn{2}{|r|}{ Sanjou \& Nezu (2013) } & 0.1 & 0.3 & 0.12 & 0.053 & 0.33 & 0.177 & 0.23 & & 0.014 \\
\hline
\end{tabular}

\section{Impact of the geometrical aspect ratio $W / L$}

The present section focuses on the impact of the geometrical aspect ratio of the cavity $W / L$ on the mass exchange processes and mass exchange coefficient from the main stream to the cavity. The first subsection presents the mean velocity fields corresponding to the 5 aspect ratios of the cavity considered in Series 1 in Table 1 then the second subsection shows the time evolution of the 2D maps of passive scalar concentration in the cavity, and finally, the third subsection estimates the passive scalar exchange coefficients.

\subsection{D time-averaged velocity fields}

In order to help understanding the mass exchange processes in the next sub-section, Fig.3 first plots the time-averaged velocity field measured through 2D PIV (as described above) at the elevation $z / h=0.71$ for the 5 configurations from Series 1 in Table 1 . To do so, the camera used to record the particle motion is placed above the cavity, the sampling frequency is reduced to $12.2 \mathrm{~Hz}$ and the spatial resolution to about $1 \mathrm{~mm}$ per pixel (see Mignot et al., 2017 for more details).

For a square cavity $(W / L=1)$, Fig.3 reveals that a single recirculation cell forms and occupies the whole available space in the cavity region, as previously shown for instance by Booij (1989), Kimura and Hosoda (1997) or Mizumura and Yamasaka (2002). The typical velocity magnitude of the recirculation cell equals about $0.025 \mathrm{~m} / \mathrm{s}$, that is about $0.15 U_{b}$ (as for Tuna et al., 2013, for a slightly longer cavity $W$ ). For a slightly wider cavity $(W / L=2)$, a second cell appears but remains confined in the downstream-external corner $(x / L \approx 1, y / L \approx 2)$. As the width of the cavity $W$ increases, the size of the second cell increases while the size of the first cell remains almost constant. For geometries with higher aspect ratios $(W / L>4)$, the flow in the second cell becomes $3 \mathrm{D}$ and particularly complex (not detailed herein). Note that the streamlines remain unclosed due to limited but still existing vertical velocities. 
For the purpose of the present paper, a major aspect of the velocity field is the velocity magnitude along the interface (at $y=0$ ) where mass exchanges occur through turbulent diffusion. This velocity slightly decreases for increasing $W / L$ values: the typical velocity in the center of the interface $(y=0$ $\& x / L \approx 0.5$ ) equals $0.0275 \mathrm{~m} / \mathrm{s}$ for $W / L=1$, and decreases to $0.0225 \mathrm{~m} / \mathrm{s}$ for $W / L=2-3$ and finally to $0.0175 \mathrm{~m} / \mathrm{s}$ for $W / L=4-5$. Thus the velocity gradient across the interface tends to increase, as $W / L$ increases, from about $\Delta U \approx 0.167-0.0275 \approx 0.14 \mathrm{~m} / \mathrm{s}$ for $W / L=1$ to $\Delta U \approx 0.166-0.0175 \approx 0.15 \mathrm{~m} / \mathrm{s}$ for $W / L=4-5$. This corresponds to an increase of velocity gradient limited to about $7 \%$. This provides a first hint that the exchange capacity should not vary much as $W / L$ varies from 1 to 5 . 

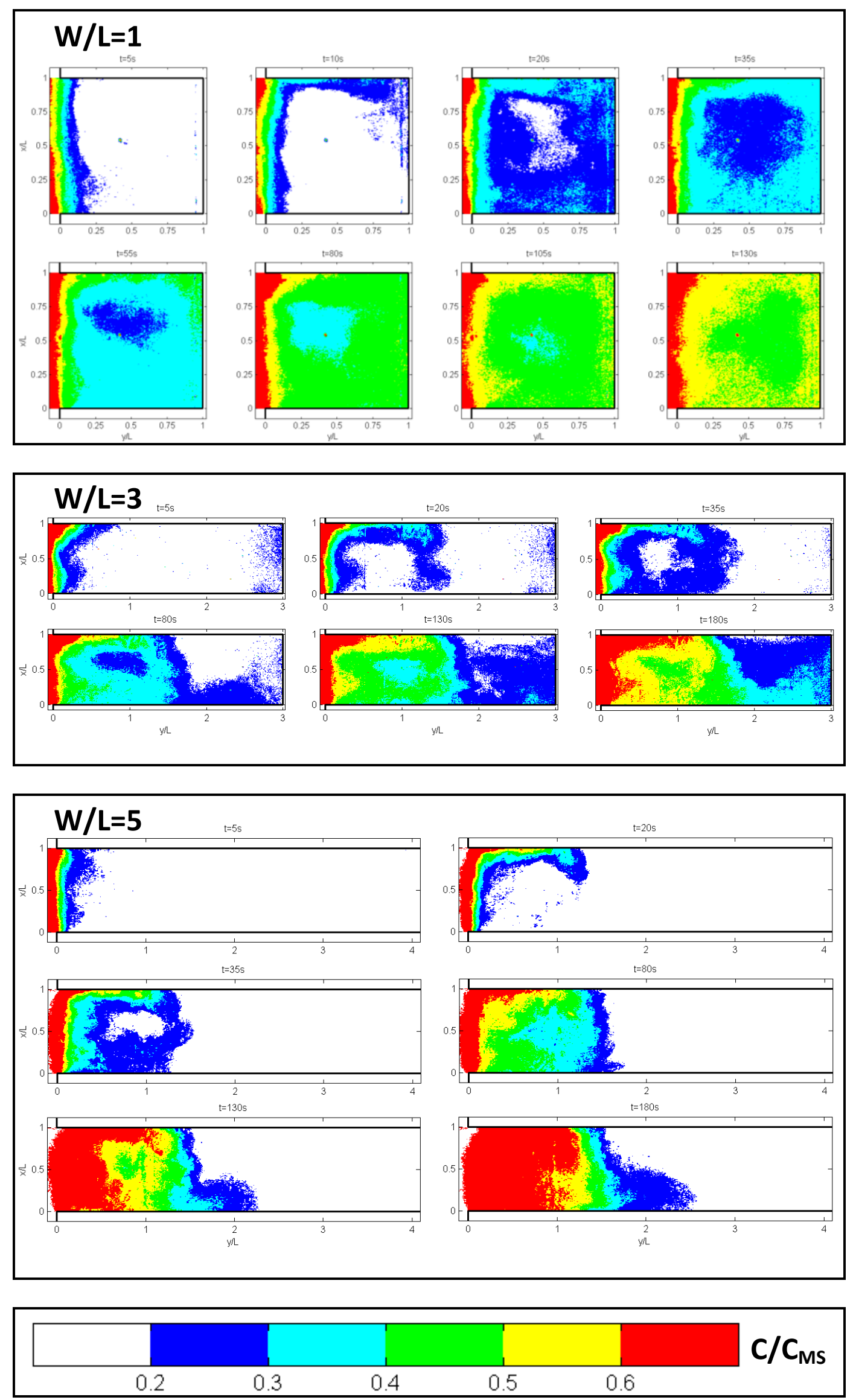

Figure 2. Time-evolution of dye concentration maps for configurations with $W / L=1,3$ and 5 . 


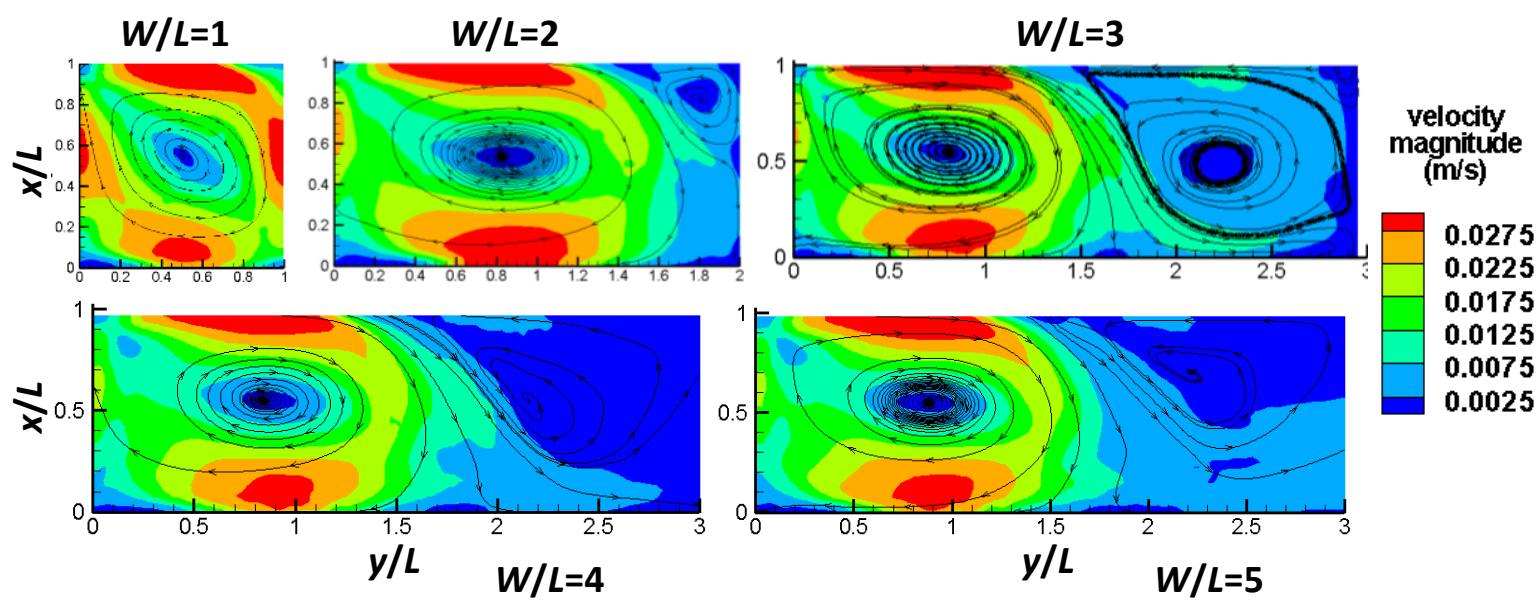

Figure 3. Time-averaged $2 D$ velocity fields measured for the 5 flow configurations of Series 1, taken from Mignot et al., 2017.

\subsection{Concentration maps}

Fig. 2 plots the concentration maps measured through the dye release method, at selected times, for three configurations from Series 1: $W / L=1,3$ and 5, while Fig.4 sketches the physical processes involved in the mass exchange from the main flow to the cavity and within the cavity. At initial times (steps 1 to 3 ), the exchange processes are similar for all $W / L$ configurations while for step 4, the processes differ for narrow (small $W / L$ ) and wide (high $W / L$ ) cavities.

Step 1. Dye enters in the cavity through turbulent diffusion in the mixing layer (arrows A on Fig.4). At time $t=5 \mathrm{~s}$ (Fig.2), dye is already present on the cavity side $(y>0)$ all along the interface (for $x / L=0$ to 1$)$. This turbulent diffusion process continues during the whole experiment.

Step 2. Dye entering the cavity during step 1 is transported through advection by the first recirculation cell of the cavity (arrow B, see Fig.4) towards the downstream corner $(x / L=1, y=0)$. This low-velocity region (see Fig.3) thus acts as a storage region where a high concentration is measured during the whole experiment for all geometries.

Step 3. The dye accumulating near the downstream corner of the cavity continues to be transported through advection by the first cell (arrow B) (i) along the downstream wall (along $x / L=1$ ), (ii) along the end wall $(y / L=1)$ for $W / L=1$ geometry and along the end of the first cell (at $y / L \approx 1.5-1.7$ ) for the wider geometries $(W / L=3$ and 5 , see for instance $t=20$ s for $W / L=3$ geometry), and (iii) along the upstream wall $(x=0)$ back to the interface region $(y \approx 0)$.

Step 4. This step only applies to cavities with two cells ( $W / L=3$ and 5) for which part of the scalar reaching the upstream wall $(x=0)$ in step 3 is transferred to the second cell at the saddle point 
$(y / L \approx 1.5$ to 1.7 and $x=0)$ through arrow D. The dye that enters the second cell is then advected in a counterclockwise motion (arrow $\mathrm{B}_{2}$ ) and finally joins the first cell along the downstream wall (at $y / L \approx 2, x / L=1)$.

Step 5. In the meantime of steps 3 and 4, turbulent diffusion (arrows $\mathrm{C}$ and $\mathrm{C}_{2}$ ) occurs from the outer layer of each cell where the concentration is high to the center of the cells where the concentration is low. Dye then reaches the center of the first cell at $t=20 \mathrm{~s}$ to $35 \mathrm{~s}$ for $W / L=1$ and at $t=35 \mathrm{~s}$ to $80 \mathrm{~s}$ for $W / L=3$ and 5 .

When comparing the scalar fields for the three geometries, it appears that at a given time, the dye distribution is much more homogenized in the square cavity than in the two wider ones (see for instance at $\mathrm{t}=80 \mathrm{~s})$. Two cumulative reasons can be listed for this effect. First, the time for the dye to rotate along the outer layer of the first cell and reach the upstream corner of the cavity $(x \approx y \approx 0)$ is much higher for wide cavities than for the narrow one (see for instance the three concentration maps at $t=20 \mathrm{~s}$ ); this is because (i) the width (along $y$ axis) of the first cell is much shorter for the narrow cavity than for the wider ones and (ii) the typical velocity in the first cell is slightly higher for the narrow cavity than in the wider ones (see Fig.3). The second reason is that for the wide cavities ( $W / L$ equal to 3 and 5), part of the dye from the first cell is transferred to the second cell.

Moreover, it appears that the exchange of scalar from the first cell towards the second cell is much more efficient for $W / L=3$, for which the second cell occupies the whole available space for $x / L>1.7$ than for $W / L=5$ for which the flow is 3-dimensional and complex (not shown here).
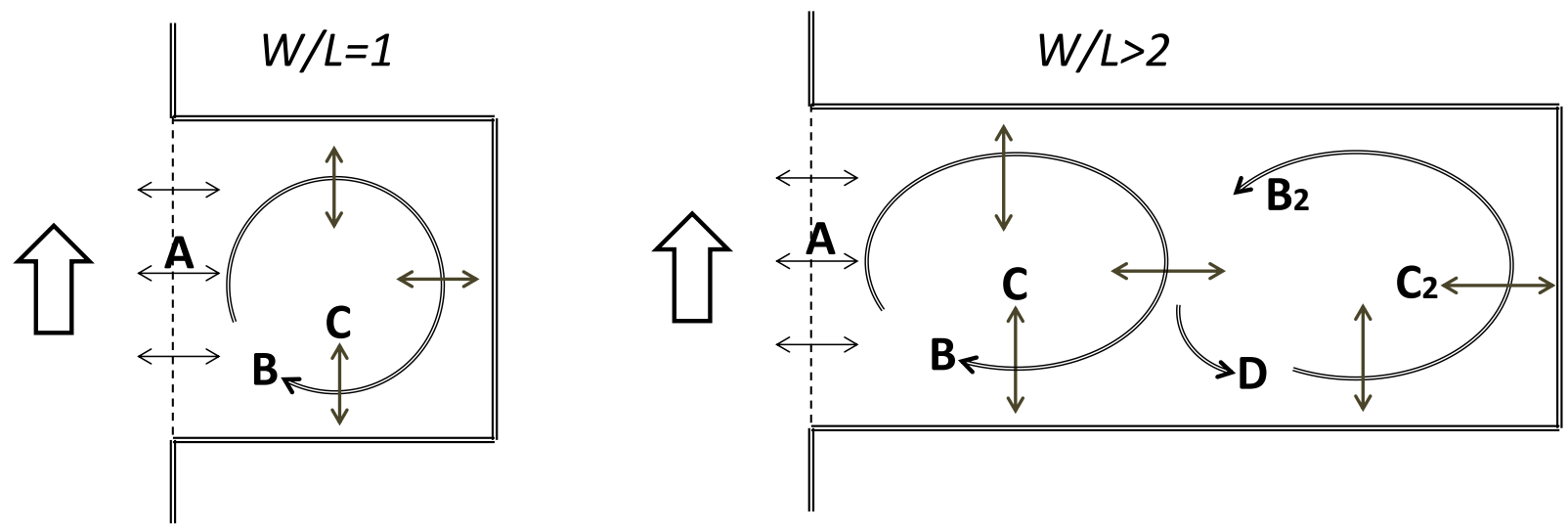

Figure 4. Scheme of the passive scalar transport processes for a cavity with a single cell (such as $W / L=1$ ) and two cells $(W / L>2)$.

\subsection{Exchange coefficients}

Following the methodology applied by Uijttewaal et al. (2001) and Weitbrecht et al. (2008), the mean concentration $C_{c}(t)$ in Eq.6 (dye release method) is obtained by spatially averaging at each 
time the concentration maps such as plotted in Fig.2 over the cavity area. Then, the relation $\left[C_{\mathrm{MS}^{-}}\right.$ $\left.C_{\mathrm{C}}(t)\right] / C_{\mathrm{MS}}=\mathrm{f}\left(t U_{b} / W\right)$ is plotted in Fig.5. According to Eq.6, the slope of the curve should be equal to $-k / \ln (10)$, permitting to estimate the $k_{\text {dye }}$ coefficient. This figure confirms that, at initial time, $k_{\text {dye }}$ is similar for the three cavities with increasing aspect ratio. Nevertheless, it appears that the absolute value of the slope decreases with time (as for Uijttewaal et al., 2001 or Sanjou and Nezu, 2013). According to Uijttewaal et al. (2001), the fact that the measured data departs from the linear curve is due to additional phenomena within the cavity such as "exchange with the center of the gyre" "where the mixing layer does not penetrate"; this exchange is "governed by small scale turbulent motion and is therefore slower". This might be true is the narrow cavities considered by these authors $(\mathrm{W} / \mathrm{L}=0.3 \& 0.7)$. However, in the present longer cavities $(\mathrm{W} / \mathrm{L} \geq 1)$, we believe that this departure is mostly due to the uncertainty associated with the dye release method, detailed in section 1.3. It is thus difficult to estimate a constant $k$ value from such plots.

The exchange coefficients can alternatively be obtained through the transverse velocity method (Eqs.8 and 2). The right-hand-side term of Eq.6 is then added to Fig.5 as solid lines for the three configurations using the $k$ values obtained through the transverse velocity method $\left(k_{\mathrm{veloc}}\right)$. The agreement between the spatially averaged concentrations (symbols) and the theoretical curve based on $k_{\text {veloc }}$ (solid lines) appears to be fair over the initial time but, as indicated above and reported in the literature, the measured concentration rapidly departs from this straight line (typically for $t>50$ s). To summarize, the dye release method gives a nice overview of the processes involved in the dye transport across the mixing layer and within the cavity but estimations of exchange coefficients based on this method must be taken with caution. Consequently only data from the transverse velocity method will be used in the following section in order to assess the impact of the non-dimensional parameters on the exchange coefficient. 


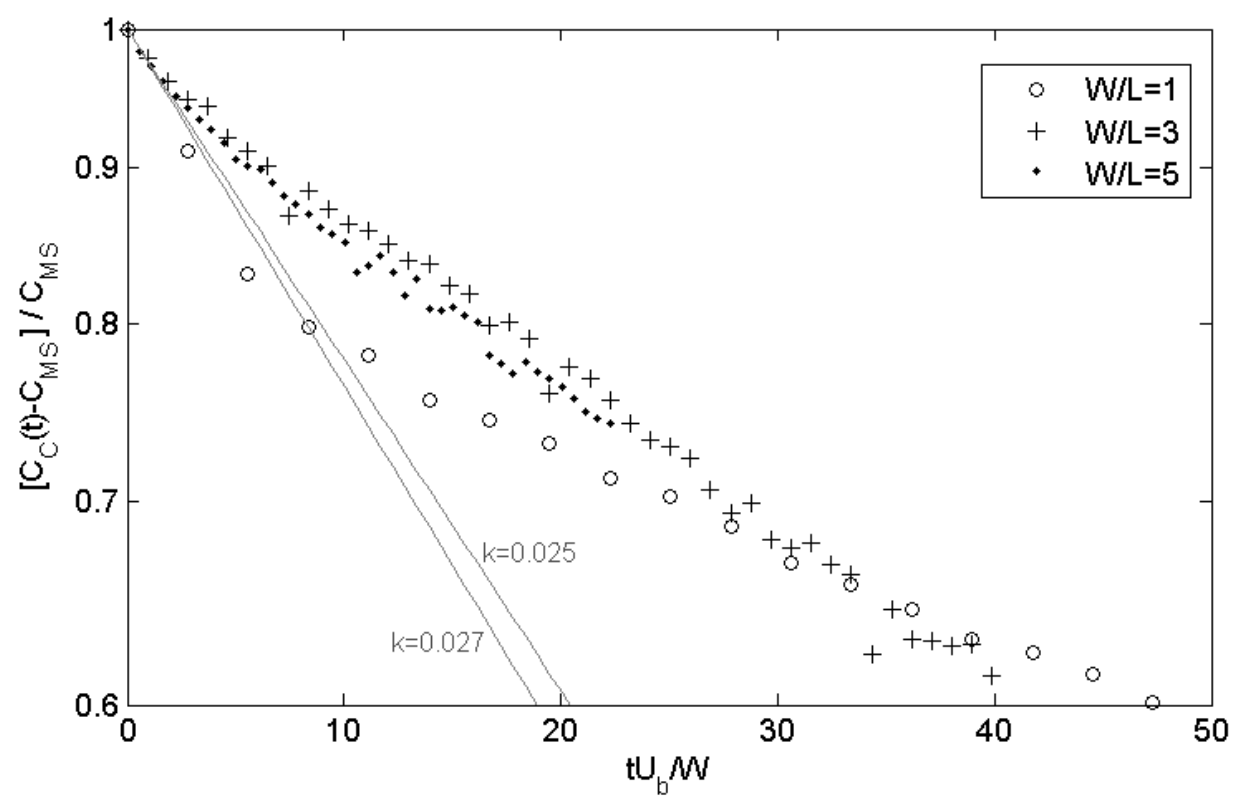

Figure 5. Time-evolution of spatially averaged dye concentration in the cavity for $W / L=1,3$ and 5 in Series 1 (symbols) along with the curves corresponding to the right hand side of $E q .6: y=\exp \left(-k U_{b} t / W\right)$ for $W / L=1,3$ and 5 with two $k$ values typical of $k_{\text {veloc }}$ measured for Series 1 in Table 1: $k=0.025$ and 0.027 (solid grey lines).

Regarding the influence of the geometrical aspect ratio of the cavity on the exchange coefficient, Table 1 reveals that $k_{\text {veloc }}$ hardly evolves as a function of $W / L$ as anticipated in section 2.1 when analyzing the 2D velocity fields. Fig.6 plots the distribution of the mean absolute transverse velocity at the measured elevation $(z / h=0.71)$ along the main stream / cavity interface, which averaged value equals $k_{\text {veloc }}$ as in Eq.8. The curves appear to be similar for all aspect ratio of the cavity and qualitatively in agreement with measurements from Tuna et al. (2013) (in their figure 14): $\overline{\mid v(x)} \mid$ is minimum near the upstream corner of the cavity and increases towards the downstream corner with a first maximum obtained near $x / L=0.7$ and a second one at the downstream corner $(x / L=1)$.

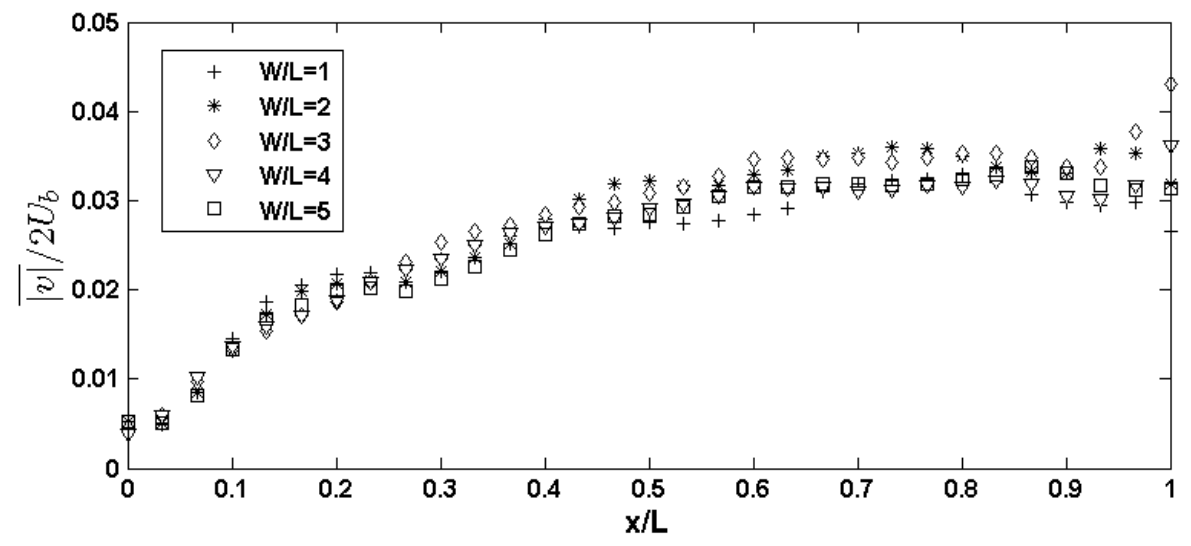

Figure 6. Mean absolute transverse velocity component measured along the interface $(y=0)$ between the main stream and the cavity for five values of $W / L$. 


\section{Impact of the two other parameters: $R e$ and $h / L$}

The impact of the main stream Reynolds number Re on the exchange coefficient is estimated through the analysis of $k_{\text {veloc }}$ values (Eq.8) for all configurations from Series 2 (Table 1) with constant $h / L$ and $W / L$ parameters. These estimates are plotted in Fig.7a. Similarly, the impact of $h / L$ on the exchange coefficient is estimated through the analysis of all configurations from Series 3 (Table 1), with constant $W / L$ but varying Re, and are plotted in Fig.7b.
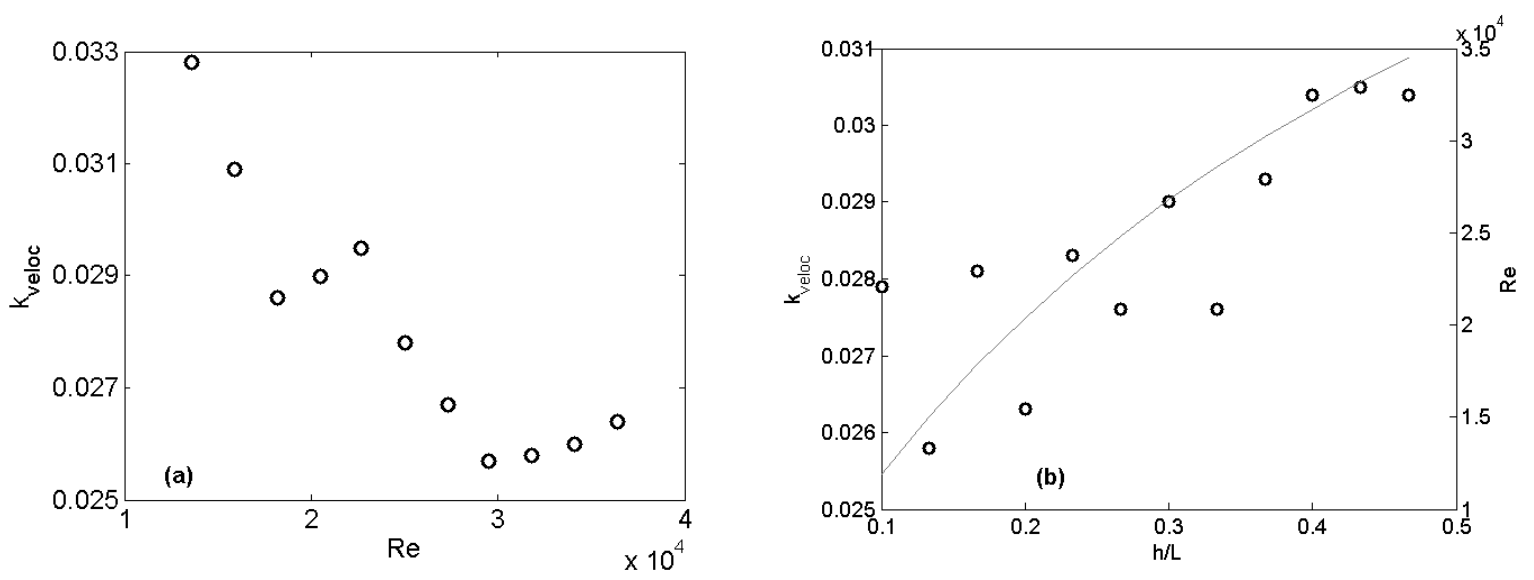

Figure 7. Evolution of $k_{\text {veloc }}$ coefficient from Series 2 as a function of Re with fixed values of $h / L(a)$ and from Series 3 as a function of h/L (symbols) with Reynolds numbers (solid line) increasing simultaneously (b).

Fig. 7 a reveals that as Re increases from $1.4 \times 10^{4}$ to $3.6 \times 10^{4}, k$ decreases by about $20 \%$. On the other hand, Fig. $7 b$ reveals that as both $h / L$ and Re increase, $k$ increases, and that as $h / L$ is multiplied by 4 , $k$ increases by about $18 \%$. Both graphs from Fig.7 allow to conclude that i) $k$ increases as Re decreases, ii) $k$ increases as $h / L$ increases and iii) in the range of variation of $h / L$ and Re considered in Series 3, the impact of $h / L$ exceeds that of Re.

To explain the reason why $k$ increases as Re decreases or $h / L$ increases, Figs. 8 a and $8 \mathrm{~b}$ plot selected profiles (from Series 2 and 3) of mean absolute transverse velocities along the mixing layer axis, in the same way as in Fig.6. This figure reveals that both $\operatorname{Re}$ and $h / L$ affect the shape of the $\overline{|v|}$ profiles: while $\overline{|v|}$ remain similar near the corners of the cavity $(x / L=0$ and 1$)$, it decreases (respectively increases) in the center region of the mixing layer as Re increases (resp. $h / L$ increases), so that the integral in Eq.8 decreases (resp. increases) and finally $k_{\text {veloc }}$ decreases (resp. increases). Two possible origins of the opposite influences of the Reynolds number and normalized water depth can be listed: 
1) the variation of these parameters affects the characteristics of the boundary layer of the inflow along the lateral wall of the upstream channel $(x<0 \& y=0)$ which, when reaching the cavity, in turn affects the development of the mixing layer.

2) such variation does not affect the inflow (upstream from the cavity) but directly modifies the development of the mixing layer at the interface between the cavity and the mean flow.

To investigate the validity of each of these two assumptions, Figs.9a and 9b plot, for Series 2 and 3 respectively, the transverse (along $y$ ) profiles of mean streamwise (along $x$ ) velocity at the elevation $z / h=0.71$ at $x / L=0.1$, i.e. near the upstream corner of the cavity, which is assumed to be representative of the lateral boundary layer of the inflow (for $x<0$ ). On the one hand, Fig.9a reveals that the Reynolds number Re hardly affects the mean streamwise velocity profile on the main stream side $(y<0)$, which discards assumption $\# 1$. On the other hand, Fig.9b indicates that the combined effects of $h / L$ and Re slightly affects the shape of the transverse boundary layer of the incoming flow: as $h / L$ and Re increase, the maximum normalized streamwise velocity magnitude increases (see the arrow in Fig.9b). Contrary to the previous case, this result does not completely discard assumption \#1.

Moreover, Figs.10a and 10b plot, for Series 2 and 3 respectively, the streamwise evolution of the mixing layer width $\delta$ computed as:

$$
\delta(x)=\frac{\Delta U(x)}{|\partial U(x) / \partial y|_{\max }}
$$

with $\Delta \mathrm{U}$ the difference of outer velocities (between the main stream and the cavity) at a given $x$ axis and the denominator is the maximum transverse (along $y$ ) gradient of mean streamwise (along $x$ ) velocity. Note that the estimation of $\delta$ for $x / L<0.1$ is uncertain due to the complexity of the velocity field in the region surrounding the stagnation point around the upstream corner of the cavity. First, it appears from Figs.10a and 10b that the mixing layer width near the upstream corner of the cavity $(x / L \approx 0.05-0.1)$ remains similar $(\delta / L \approx 0.09)$ for all configurations. This result confirms the observation from Fig.9a (respectively Fig.9b) that, in the ranges considered in Table 1, the Reynolds number (respectively the Reynolds number and the normalized water depth) does not affect (respectively have a limited impact) on the upstream boundary layer development.

Oppositely, Figs.10a and 10b reveal that further downstream $(x / L>0.1)$, the mixing layer width increasing rate is strongly dependent on both parameters $(\operatorname{Re}$ and $h / L)$ : it decreases as Re increases in Fig.10a and as $h / L$ and Re decrease in Fig.10b. This increasing rate, noted $\alpha$ herein, can be estimated through the following classical equation, simplified for the present case assuming that the 
typical outer velocity (noted with a tilde) on the mean flow side equals about $U_{\mathrm{b}}$ and is nil on the cavity side:

$$
\frac{d \delta}{d x}=\alpha \frac{\Delta \widetilde{U}}{\widetilde{U}_{c}} \approx \alpha \frac{U_{b}}{U_{b} / 2} \approx 2 \alpha
$$

with $\Delta \widetilde{U}$ the difference between the typical outer velocities on each side, and $\widetilde{U}_{c}$ their average value. Tendency curves are added as grey solid lines on Figs.10a and 10b for the minimum and maximum $\alpha$ values reported by Pope (2000) based on a review of the literature. The figures confirm that $\alpha$ is included in this range.

The fact that $\alpha$ decreases as Re increases (Fig.10a) and as $h / L$ and Re decrease (Fig.10b) gives credit to hypothesis \#2 for both series (2 and 3): Re and $h / L$ directly affect the mixing layer development along the main stream / cavity interface and this is responsible for the decreasing exchange coefficients $k$ values as Re increases or as $h / L$ decreases (Table 1 and Fig.7). Note that a decrease of $\alpha$ and thus of $\delta$ indicates that the typical sizes of the coherent turbulent structures advected along the mixing layer are of reduced transverse (along y) extent (see Mignot et al., 2016) and thus that the efficiency of passive scalar transfer through turbulent diffusion across the interface also decreases. The fact that decreasing the water depth tends to decrease the horizontal extension of the coherent turbulent structures was recently observed by Han et al. (2017).
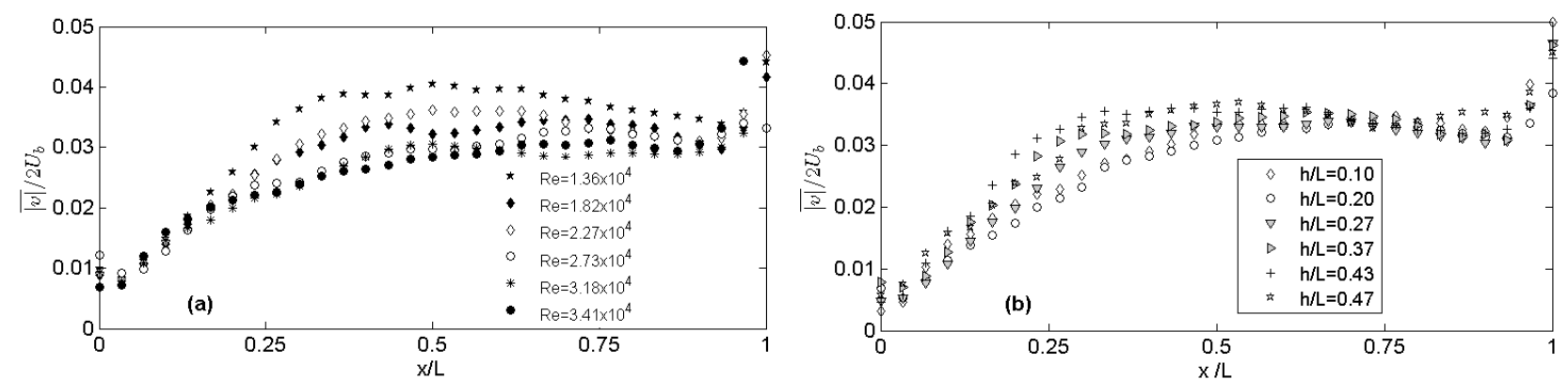

Figure 8: Streamwise evolution of the absolute value of the normalized transverse velocity component at the interface between the main stream and the cavity $(y=0)$ for Series 2 (selected configurations, a) and Series 3 (selected configurations, $b$ )
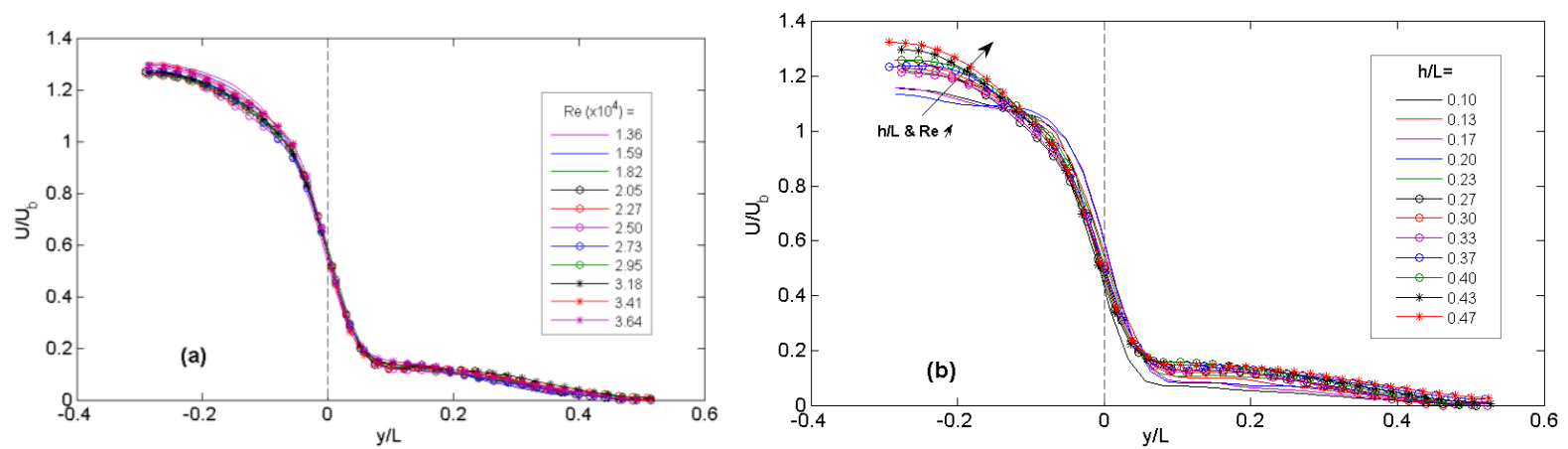
Figure 9 Transverse (along $y$ ) profiles of normalized mean streamwise (along $x$ ) velocity $U$ measured across the cavity $(y>0) /$ main stream $(y<0)$ interface at $z / h=0.71$ and $x / L=0.1$ for series $2(a)$ and series $3(b)$ configurations from Table 1.
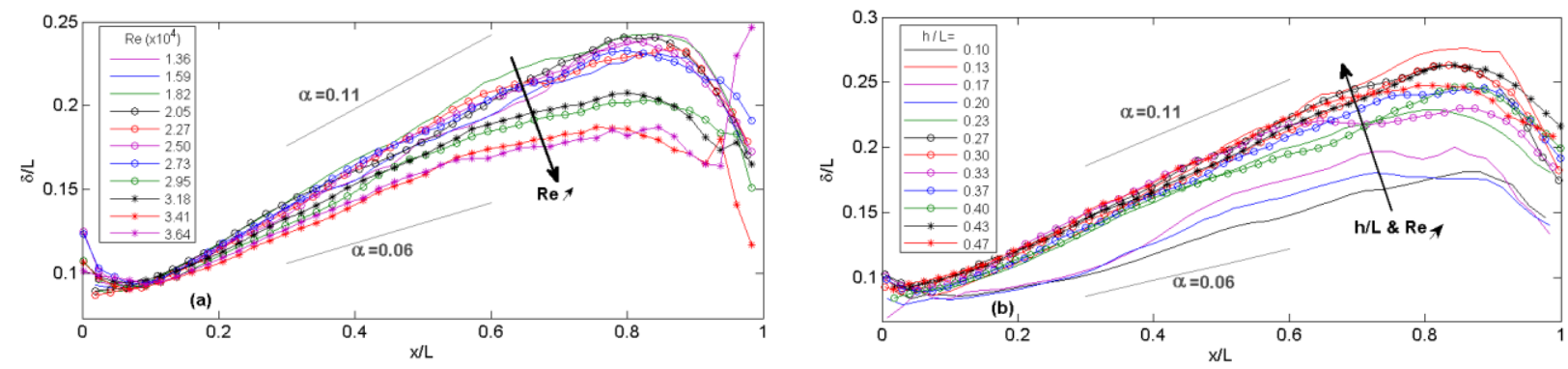

Figure 10 Streamwise evolution of the normalized mixing layer width $\delta / L$ for series 2 (a) and series 3 (b) configurations from see Table 1 along with tendency curves corresponding to Eq.10 with $\alpha=0.06$ and 0.11 (solid grey lines).

\section{Discussions and conclusions}

4.1 Comparison with tendencies available in the literature

The sensitivity analysis performed above permitted to identify the non-dimensional parameters that impact the coefficient of mass exchange between a simplified, wide $(W / L>1)$ lateral cavity and a subcritical straight open-channel flow. We showed that the geometrical aspect ratio of the cavity does not affect this coefficient, but that an increase of the normalized water depth $h / L$ or a decrease of the main stream Reynolds number Re tend to increase it. The aim of the present section is to compare these tendencies with the ones reported for cavities located within groyne fields, mostly by Weitbrecht et al. (2008), and in complex shape cavities and cavities located within groyne fields by Jackson et al. (2015). Note that a section in the introduction discusses on the differences between isolated lateral cavities (as considered herein) and cavities located within groyne fields. First we checked (not shown here) that the fitting curve proposed by Weitbrecht et al. (2008), which relates the exchange coefficient to the so-called morphometric parameter $R_{\mathrm{d}}$ does not apply to the present (Table 2) and literature (Table 1) cavity data. Moreover, applying the two empirical relations proposed by Jackson et al. (2015) to the present (respectively from the literature) cavity configurations lead to a typical difference in $k$ of $42 \%$ (respectively $46 \%$ ) using their equation 8 and $52 \%$ (respectively 29\%) using their equation 9 . None of these empirical relations appears to be appropriate to the simplified lateral cavity configuration.

Moreover, regarding the qualitative evolution of $k$ :

-In Series 1 (Table 1), the variation of the $W / L$ aspect ratio of the cavity appears not to affect the value of $k$, while Weitbrecht et al. (2008) and Jackson et al. (2015) investigations indicate that $k$ decreases as $W / L$ increases. 
-In Series 2, $k$ appears to decrease as the bulk velocity of the mean flow $U_{b}$ is increased, in agreement with equation (8) from Jackson et al. (2015), but not with equation (9) from Jackson et al. (2015) and the work from Weitbrecht et al. (2008), both of which predict no effect of $U_{b}$ on $k$.

-In Series 3, $k$ appears to increase as the water depth increases while Weitbrecht et al. (2008) and Jackson et al. (2015) investigations predict that $k$ decreases as the water depth increases.

These unsatisfactory agreements with the tendencies proposed in the literature confirm the statement made in the introduction that groyne and cavity flows essentially differ in terms of hydrodynamics and that the processes governing the mass exchange from the main stream to the dead zones is strongly dependent on the type of storage zone. Notably, present results highlight the influence of the mixing layer development on the exchange coefficient. Yet, this mixing layer is itself considerably influenced by the upstream groynes in the groyned field cases, as shown by McCoy et al. (2008)

\subsection{Conclusions}

The main findings of the present work regarding mass exchange between a straight open-channel flow and its adjacent rectangular lateral cavity are finally listed below:

- The processes involved in the introduction and spreading of passive scalar in the cavity rely on the turbulent diffusion across the interface, the advection by the mean flow in the cavity and the turbulent diffusion within each recirculating cell and from one cell to the neighboring one (when existing).

- The use of the dye release method, useful for qualitative exchange analyses, should be taken with caution for estimating the mass exchange coefficient, even though its use during the very first stage of the mass exchange appears to be valid. On the other hand, the transverse velocity method should also be taken with caution as it is based on velocity measurements rather than concentrations; also, it is representative of the exchange processes only at early stages of mixing process, i.e. when the transfer is regulated by the dynamics of the shear layer.

- The dimensional analysis applied to this exchange coefficient brings to light three nondimensional parameters. Their impact on the exchange coefficient can be summarized as:

* the geometric aspect ratio of the cavity does not affect the exchange coefficient, at least as long as $W / L$ remains larger than 1 ;

* increasing the normalized water depth tends to increase the exchange coefficient;

* increasing the Reynolds number of the main stream tends to decrease the exchange coefficient.

We showed that, in the present range of variation of these parameters, the boundary layer of the inflow is almost not affected by the variation of these parameters, but that the parameters directly 
affect the characteristics of the mixing layer at the interface between both flow regions, which in turn affect the mass exchange capacities between the main channel and the cavity.

\section{Acknowledgments}

This work was supported by ECOS-Conicyt C11E02 and Fondecyt 1130940 grants. We also acknowledge the funding from Conicyt/Fondap grant 15110017 and the $\mathrm{PhD}$ grant of W. Cai from the Chinese Scholarship Council.

\section{References}

Altai, W. \& Chu, V. H. 1997. Retention time in a recirculating flow. $27^{\text {th }}$ congress of IAHR, 10-15 August 1997, San Francisco, USA, 9-14.

Argerich, A., E. Marti, F. Sabater, R. Haggerty, \& Ribot M. 2011. Influence of transient storage on stream nutrient uptake based on substrata manipulation. Aquatic Sciences, 73, 365-376, doi:10.0007/s00027-011-0184-9.

Booij, R. 1989. Exchange of mass in harbors. $23^{\text {rd }}$ congress of IAHR, 21-25 August 1989, Ottawa, Canada, D69-D74.

Caton, F., Britter, R.E. \& Dalziel, S. 2003. Dispersion mechanism in a street Canyon. Atmospheric Environment, 37, 693-702.

Ensign, S. H., \& Doyle, M.W. 2005. In-channel transient storage and associated nutrient retention: Evidence from experimental manipulations. Limnology and Oceanography, 50(6), 1740-1751.

Erpicum S., Meile T., Dewals B., Pirotton, M. \& Schleiss, A. 2009. 2D numerical flow modeling in a macro-rough channel. Int. J. for Numerical Methods in Fluids, 61, 1227-1246.

Han, L., Mignot, E. \& Riviere, N. 2017. Shallow mixing layer downstream a sudden expansion, Journal of Hydraulic Engineering, accepted for publication.

Jackson, T.R., Haggerty, R., Apte S.V. \& O’Connor, B.L. 2013. A mean residence time relationship for lateral cavities in gravel-bed rivers and streams: Incorporating streambed roughness and cavity shape. Water Resources Research, 49, 3642-3650.

Jackson, T.R., Apte S.V., Haggerty R. \& Budwig, R. 2015. Flow structure and mean residence times of lateral cavities in open channel flows: influence of bed roughness and shape. Environmental Fluid Mechanics, 15 (5), 1069-1100

Jahanmiri, M. 2011. Particle image velocimetry: Fundamentals and its application. in Report 2011:03, ISSN 1652-8549, Chalmers University of Technology, Goteborg, Sweden.

Karimpour, S., \& Chu, V. H. 2015. Mixing in shallow waters in high Froude number. 22nd Canadian Hydrotechnical Conference - Water for Sustainable Development: Coping with Climate and Environmental Changes. Montreal, Canada, April 29 - May 2, 2015.

Kimura, I., \& Hosoda, T. 1997. Fundamental properties of flows in open channels with dead zone. Journal of Hydraulic Engineering, 123(2), 98-107.

Langendoen, E.J., Kranenburg, C. \& Booij, R. 1994. Flow patterns and exchange of matter in tidal harbours. Journal of Hydraulic Research, 32(2), 259-270. 
Lecoz, J., Michalkova, M., Hauet, A., Comaj, M., Dramais, G., Holubova, K., Piegay, H. \& Paquier, A. 2010. Morphodynamics of the exit of a cutoff meander: experimental findings from field and laboratory studies. Earth Surface Processes and Landforms, 35, 249-261.

Li, C.W. \& Gu, J. 2002. 3D layered-integrated modelling of mass exchange in semi-enclosed water bodies. Journal of Hydraulic Research, 39(4), 403-411.

Li, C.W. \& Ip, K.W. 1999. Residence time in semi-enclosed water bodies. Civil and Environmental Engineering Conference, 8-12 November 1999, Bangkok, Thailand, VI.13-VI.16

McCoy, A., Constantinescu, G. \& Weber, L.J. 2008. Numerical investigation of flow hydrodynamics in a channel with a series of groynes. Journal of Hydraulic Engineering, 134(2), 157-172.

Markides, C.N., Fokaides, P.A. \& Neophytou, M.K. 2010. Flow and exchange processes in homogeneous urban street canyon geometries: an experimental study using particle image velocimetry. $9^{\text {th }}$ HSTAM International Congress on Mechanics, Limassol, Cyprius.

Meile, T., Boillat, J-L. \& Schleiss, A.J. 2011. Water-surface oscillations in channels with axi-symmetric cavities. Journal of Hydraulic Research, 49(1), 73-81.

Mignot, E., Zeng, C., Dominguez, G., Li, C.-W., Rivière, N. \& Bazin, P.-H. 2013. Impact of topographic obstacles on the discharge distribution in open-channel bifurcations. Journal of Hydrology, 494(28), 10-19.

Mignot, E., Doppler, D., Riviere, N., Vinkovic, I., Gence, J. \& Simoens S. 2014. Analysis of flow separation using a local frame-axis: application to the open-channel bifurcation. Journal of Hydraulic Engineering, 140, $280-290$.

Mignot, E., Cai, W., Launay, G., Riviere, N. \& Escauriaza, C. 2016. Coherent turbulent structures at the mixinginterface of a square open-channel lateral cavity. Physics of Fluids, 28, 045104.

Mignot, E., Cai, W. \& Riviere, N. 2017. Measurement of the flow pattern in open-channel lateral cavities with increasing aspect ratio, submitted to Journal of Hydraulic Engineering.

Mizumura, K. \& Yamasaka, M. 2002. Flow in open channel embayments. 128(12), 1098-1101.

Muto, Y., Imamoto, H. \& Ishigaki, T. 2000. Velocity measurements in a straight open channel with a rectangular embayment. Proc. $12^{\text {th }}$ APD-IAHR, Bangkok, Thailand, 353.

Pope, S. 2000. Turbulent flows, Cambridge University Press.

Salizzoni, P., Soulhac, L. \& Mejean, P. 2009. Street canyon ventilation and atmospheric turbulence. Atmospheric Environment, 43 (32), 5056-5067.

Sanjou, M. \& Nezu, I. 2013. Hydrodynamic characteristics and related mass-transfer properties in open-channel flows with rectangular embayment zone. Environmental Fluid Mechanics, 13(6), 527-555.

Tuna, B.A., Tinar, E. \& Rockwell, D. 2013. Shallow flow past a cavity: globally coupled oscillations as a function of depth. Experiments in Fluids, 54, 1586.

Tuna, B.A. \& Rockwell, D. 2014. Self-sustained oscillations of shallow flow past sequential cavities. Journal of Fluid Mechanics, 758, 655-685.

Uijtewaal, W., Lehmann, D. \& van Mazijk, A. 2001. Exchange processes between a river and its groyne fields: model experiments. Journal of Hydraulic Engineering, 127(11), 928-936.

Valentine, E.M. \& Wood, I.R. 1977. Longitudinal dispersion with dead zones. Journal of Hydraulic Division, ASCE, 103(9), 975-990.

Weitbrecht, V., Socolofsky, S.A. \& Jirka, G.H. 2008. Experiments on mass exchange between groin fields and main stream rivers. Journal of Hydraulic Engineering, 134(2), 173-183. 